\section{Public Health Genomics}

Public Health Genomics 2015;18:1-10

DOI: $10.1159 / 000364993$
Received: January 9, 2014

Accepted after revision: June 3, 2014 Published online: July 25, 2014

\title{
Extending the Surrogacy Analogy: Applying the Advance Directive Model to Biobanks
}

\author{
Stephanie Solomon ${ }^{\mathrm{a}}$ Ann Mongoven ${ }^{\mathrm{b}, \mathrm{c}}$

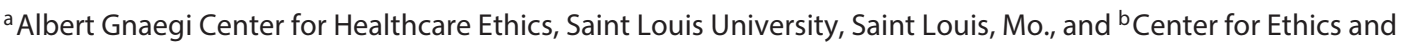 \\ Humanities in the Life Sciences, and ' Department of Pediatrics and Human Development, College of Human \\ Medicine, Michigan State University, East Lansing, Mich., USA
}

\section{Key Words}

Advance directives - Biobanking $\cdot$ Community

engagement $\cdot$ Informed consent $\cdot$ Surrogacy

\begin{abstract}
Biobank donors and biobank governance face a conceptual challenge akin to clinical patients and their designated surrogate decision-makers, the necessity of making decisions and policies now that must be implemented under future unknown circumstances. We propose that biobanks take advantage of this parallel to learn lessons from the historical trajectory of advance directives and develop models analogous to current 'best practice' advance directives such as Values Histories and The Five Wishes. We suggest how such models could improve biobanks' engagement both with communities and with individual donors by being more honest about the limits of current disclosure and eliciting information to ensure the protection of donor interests more robustly through time than current 'informed consent' processes in biobanking.

(c) 2014 S. Karger AG, Basel
\end{abstract}

S.S. and A.M. contributed equally to this work.

\section{Introduction}

Increasing efforts are underway to obtain 'informed consent' from donors of tissue samples to biobanks. In a previous essay, we argued against the unquestioned presumption of an analogy between informed consent for research and informed consent for biobanking [1]. In spite of arguments to the contrary, we still believe that there is a significant disanalogy between the 2 contexts [2]. A key ethical goal in research consent processes is to provide adequate information about the study so that recruits can weigh potential risks and benefits of participation. However, in biobank recruitment, such adequate disclosure necessarily cannot occur because biobanks are designed to enable future unknown studies rather than disclose the reality of present ones. This is the case in spite of the fact that biobank participants may prefer to be asked for their informed consent [3]. Our argument is not that it cannot or should not be requested, but that it fundamentally cannot be provided.

That is true even for biobanks that best approximate the unreachable asymptote of true informed consent, such as those administered by disease advocacy groups, e.g. the Genetic Alliance, an umbrella organization of North American rare genetic disease advocacy groups.

Stephanie Solomon

Albert Gnaegi Center for Healthcare Ethics, Saint Louis University

Salus Center, 3545 Lafayette Avenue, Suite 505

Saint Louis, MO 63104-1314 (USA)

E-Mail solomon2@slu.edu 
Genetic Alliance aims to develop a patient-centric research network. Its biobank provides a variety of pathways for donors of biosamples, or of health data, to engage in establishing the goals and parameters for the use of their sample. The 'Reg4All' registration system uses an internet-based system to allow donors to make choices such as whether their sample must be de-identified and what kinds of researchers may use it. Reg4All also allows continuous revisiting of these decisions through time [4].

We consider this model an exception that proves the rule. It presupposes a high level of commitment and knowledge from both the donors and the administrators of the biobank, both at the outset and through time, which is not representative of the level of commitment found in the general nondiseased population or in general public biobank administration. Constituents of Genetic Alliance have a special motivation for such ongoing engagement, since they know their own health or that of their children may be directly impacted by the research. The biobank was founded by families with rare genetic conditions, so the administrators identify strongly with the interests of donors. In terms of the advance directive analogy, participants in Genetic Alliance know the condition that most endangers their medical future and its characteristic features. They also belong to a 'family' that is both loving and competent. It would be ethically dangerous to assume the same of most donors and biobanks.

Due to the practical impossibility of true informed consent in the biobanking context, biobanks should look to alternative models of ethical engagement with their donors. Advance care planning in clinical medicine shares crucial similarities with biobanking. Like patients engaged in advance care planning, biobank donors must try to imagine what they would choose in future unknown scenarios. Biobanks, likewise, must play a surrogate role by deciding what specific research to support with donor samples in the future and how to treat those samples in future contexts, relying on information obtained at a sometimes long previous date. Focusing on the ethical role of surrogate decision-makers, we argued that clinical surrogates' obligations to become familiar with patient values and to act upon them suggest structurally analogous obligations between biobanks and their donors.

In this essay, we demonstrate the utility of this novel analogy by exploring a central aspect of the advance care planning process: advance directives, sometimes colloquially called 'living wills'. Challenges faced in the evolution of advance directives structurally parallel current challenges faced by biobanks. Contemporary advance directives that emerged out of this evolution suggest con- crete ethical models for biobanks to replace misleading 'informed consent' processes.

By recognizing their parallel challenges and learning from current approaches to advance directives, biobanks could (1) improve the transparency and integrity of the process of engaging with donors and their communities and (2) improve the interaction between the donor process and biobank decisionmaking to yield results that are more likely consonant with donor values. This shift also has implications for biobank governance and current biobank engagement practices. Although this is not the emphasis of this manuscript, we will allude to these ramifications of our proposed model.

\section{Theoretical Parallels}

The simple idea underlying advance directives is that in cases where patients cannot make choices for themselves, patients would want trusted others to make the same treatment decision they themselves would make if they could magically be given a moment of competence. Advance directives are designed to enable, to the fullest extent possible, the so-called 'substituted judgment' standard of proxy decisionmaking, whereby surrogates execute what the patient would want. When advance directives provide inconclusive evidence about what the patient would want, surrogates are expected to supplement them by invoking beneficence-oriented standards for proxy decisionmaking - 'best interest' or 'reasonable person' standards [5-7]. This hierarchy of standards is in place in most medical contexts that give cultural priority to respect for personal autonomy, such as the U.S.

In general, 2 kinds of conceptual conundrums complicate advance directives. First, since future medical circumstances are unpredictable, advance directives pose translation challenges for surrogates. What do the advance directive's general statements, or articulated desires for medical scenarios that did not arise, suggest for decisionmaking in the actual circumstances that did arise? Second, even if future medical circumstances were predictable, it is difficult to predict one's reaction to them. People's reaction to a hypothetical situation may not be the same as their reaction in the actual situation. Moreover, people's reactions may change over time, as human identity itself changes over time [8-10]. Significantly, the same 2 epistemic limits plague informed consent for biobanking: future research circumstances and the social contexts surrounding them are unpredictable, as are donors' future attitudes. 
In the clinic, these conundrums have not rendered advance directives a futile endeavor. Imperfect information about what patients would want is better than no information. Such information has proven to be important for surrogate well-being as well as for patient well-being [1113]. However, 2 consequences have resulted from the theoretical challenges of advance directives. First, there has been a quest for improved methods of advance directive articulation. Second, there has been a transformation in the understanding of substituted judgment, from a conception of it as an objective measure to a conception of it as a communicative process. Associated developments could inform biobanks' efforts to respect donor wishes in the context of similar conceptual challenges.

\section{Historical Parallels}

\section{Stage 1: Simple Advance Directives and Blanket Consent}

Advance directives were originally developed in response to cultural concerns that overtreatment in terminal or low quality-of-life cases might overburden patients and families, or violate human dignity [14]. Public attention to the 1975 Karen Quinlan case, which involved questions of whether families could limit life-sustaining treatment for a permanently comatose loved one, catalyzed the first state and national legislative efforts to give legal force to advance directives. Ultimately, these efforts resulted in the Patient Self-Determination Act of 1990, which solidified the legal standing of advance directives [14]. In response to the issues of the day, advance directives used general language to discourage overly aggressive treatment in dire circumstances, language such as avoiding 'extraordinary care'. The vagueness of the language often made it difficult to apply to specific medical circumstances (Which treatments should be considered 'extraordinary', and under what circumstances?).

Early advance directives are comparable to 'broad' or 'blanket' consent in biobanking. Blanket consent for biobanking also resorts to broad and vague language regarding donor intent: the donor gives permission to the biobank to use her sample for 'health research', or 'research in the public good'. But the biobank has no indication what kinds of research the donor considers in the public good, or how narrowly or broadly the donor interprets 'health'. The much-discussed contemporary U.S. case of the Havasupai Indians exemplifies this problem. The Havasupai never intended their broad consent to 'study the causes of behavioral/medical disorders' to allow the use of tribal specimens for research on interbreeding, schizophrenia, and ancestral origins which they found harmful and offensive [15] ${ }^{1}$.

There may be more variety among goals implied by commonly trumpeted broad potentials of biobanking for the 'common good' than is admitted among advocates. These may include: increasing the health of the overall population, improving the health of the worst off or most vulnerable, reducing health disparities, increasing fairness in healthcare access or in the benefit/burden distribution of research, enabling the most open future for children, extending human life span, increasing the cost-effectiveness of healthcare systems, etc. Although some urge a response to override these concerns for the common good, the unfortunate ethical and practical fallout from not respecting public goals far overshadows the benefits that may be achieved by ignoring them [17].

\section{Stage 2: Complex Advance Directives and Tiered Consent}

Not only did vague early advance directives prove hard to interpret, they also proved inadequate to changing social conditions. In the subsequent era of healthcare inflation, managed care and rising uninsurance, many began to worry as much about the possibility of undertreatment as of overtreatment. As concerns underlying advance directives became more complex, so too did advance directives. Patients were urged to respond to distinct imagined medical scenarios and to elaborate lists of treatments they would and would not want in such circumstances [18]. Inevitably, even the most exhaustive advance directive options did not anticipate the actual scenarios that befell patients due to the near infinite possibilities as well as evolutions in technology and care, and their sheer complexity made translation challenging for surrogates.

The movement toward more specific advance directives presages the movement from blanket toward 'tiered consent' in biobanking. In tiered consent, directives for kinds of research to be supported or avoided are included in consent documentation. Specific advance directives at-

\footnotetext{
The issues at stake in the Havasupai case go beyond the disputed interpretation of a broad consent that has garnered most public attention. Broader disconnections between cultural values of the research community and the donor communities may have made what seemed obviously fruitful research directions to one unthinkable to the other. This contrast between community research goals and academic research goals can be found widely, but is most marked in cases of aboriginal communities. For example, Taiwan's aboriginal community raised concerns that a biobanking project might inherently compromise communal senses of tribal genetic privacy, or may be conscripted by Chinese colonialist presumptions [16].
} 
tempt the precise delineation of desired treatments; analogously tiered consents in biobanking seek the precise delineation of desired research or research areas. Tiered consent choices may be based on disease groups or on kinds of research analysis to be done or avoided - for example, genetic versus non-genetic, linked data versus not, etc. Tiered consents are vulnerable to the same critique as complex advance directives: their specificity gives an illusory sense of donor power, since current tiered consent rubrics may be untranslatable to future research - while their sheer complexity places burdens on both donors and biobanks $[19,20]$. Given the unknown nature of both future health challenges and future research, tiered consent in biobanking is analogous to the young healthy patient puzzling to create highly specific medical advance directives for her distant future.

Since problems with blanket and tiered consent directly parallel problems with overly broad or overly narrow clinical advance directives, biobanks should consider resolutions achieved with advance directives to improve biobanking practices.

\section{The Solution: Shift to Values and Communicative Processes}

The pendulum swing from overly broad to overly specific advance directives failed to make them more practicable. The advance directive resolution that has emerged is marked by 2 conceptual shifts. First, there has been a shift from choosing among detailed future choices alone to encouraging patients to further articulate broader stable values that can inform treatment choices. Second, there has been a shift from thinking of advance directives as a 'legal transactional model', where a specific choice is made and this ends the communication, to a 'continuous communicative process model', where the relationship between specific choices and broader values and choices, both at once and through time, should be engaged [21].

These shifts were accomplished by the introduction of 2 new forms of advance directive communication: 'Values Histories' dialogues and subsequent streamlined tools such as The Five Wishes. We believe the conceptual shift to values and communicative processes, as well as the iterative process suggested by Values Histories and The Five Wishes, provide important lessons and guidance to existing biobanking permission processes.

\section{Values Histories}

Beginning in the early 1990s, some medical ethicists began urging the use of Values Histories. While one goal of Values Histories was to encourage prospective discussion between patients and their physicians, they were also advocated as appendages to preexisting advance directives to clarify otherwise vague language (such as 'no heroic treatment'). Values histories locate treatment options within a broader context of patient values. For example, one prominent values history first prompts the patient with terminal diagnosis to locate herself on a spectrum of value priority between quantity and quality of life. It then presents a series of possible medical interventions along a spectrum from most to least aggressive, beginning with cardiopulmonary resuscitation and moving toward options such as admission to a general hospital ward, but not a critical care unit [22]. Another popular values history instrument additionally prompts patients to discuss their level of trust in doctors, family and friends as caregivers, and describe what kinds of life moments give them joy [23].

Narrative theorists criticized the first wave of values histories for failing to explore adequately the implications of the term 'histories' [24]. People's value development is connected to the stories they tell about themselves and in which they situate themselves - narratives that are also evolving but may develop stable elements over time. Narrative values histories are also inherently communal. Narrative approaches to values histories envision the process as encouraging storytelling and feedback between the patient and the designated surrogates - who might be rendered coauthors of this narrative in unique ways in the medical future. The values history movement argued that decisionmaking authentic to a patient's overarching life and values is a more realistic, appropriate goal for advance directives than substituted judgment literally understood [9].

The values history movement shifts the primary focus of conversation to likely outcomes of possible treatment choices and their connection to patient goals, rather than to exclusive stress on treatment modalities or specific choice options. Advocates of values histories hope the conjunction of elicited values statements and hypothetical treatment decisions reduce 'translation' challenges for proxy decision-makers $[25,26]$. Embedding treatment modalities in the broader context of the patient's overall goals and life narratives changed the interpretation of hypothetical scenarios in advance directive formation. In values histories, specific scenarios are not predictions of future situations (which often cannot be predicted), but 
Table 1. The Five Wishes

\begin{tabular}{lll}
\hline Wish & Description \\
\hline 1 & 'The person I want to make care decisions when I can't.' & $\begin{array}{l}\text { - Prompts patients to choose a surrogate and define the extent of their powers. } \\
\text { - Advises patients about desirable or undesirable characteristics in surrogates. }\end{array}$ \\
\hline $\begin{array}{l}\text { 'The kind of medical care I want } \\
\text { or don't want.' }\end{array}$ & $\begin{array}{l}\text { - Provides checklists of frequently heard general values statements with items } \\
\text { that can be checked, crossed-out, or supplemented. } \\
\text { 'How comfortable I want to be.' } \\
\text { 'How I want people to treat me.' }\end{array}$ & $\begin{array}{l}\text { - Examples: priority on pain control or rejection of intention to end life. } \\
\text { 'What I want my loved ones to know.' }\end{array}$ \\
\hline
\end{tabular}

rather serve as part of exercises through which to clarify the outcome goals and values statements that will more fundamentally guide future surrogate choices.

Elements of the values history movement could redress challenges we indicated above in tiered consent. Prompting donor recruits to reflect on what overriding goals for research emerge from their life stories could help biobanks determine how to evaluate unanticipated future research modalities on their behalf. In other words, specific (or tiered) choices should still be discussed with potential biobank donors, but rather than giving the impression that these are concrete future decisions from which they can choose, they can be presented as part of a larger values conversation while emphasizing that future research will differ in as-yet unknown ways.

While values histories can provide biobanks with lessons that would improve the honesty and the accuracy of the permission process, challenges that emerged using values histories for advance directives also imply practical limits for biobanks. While values histories may be promising in terms of enabling good 'translation' on the part of surrogates, the process of working through the referent stories and hypothetical situations in order to develop them requires time and resources from all parties involved. It has proven unreasonable to expect all patients and their families to complete them. In response, promoters of values histories have begun to adapt their extensive processes into shorter, simpler decision aids for patients. The most successful of these efforts is The Five Wishes advance directive document and accompanying movement [20].

\section{The Five Wishes}

Recognized as a legal advance directive in 42 of 50 American states, The Five Wishes is a simple interactive brochure that explores 5 personal wishes, providing accompanying guidance to enable proxy decisionmaking in the medical future. Distilling down the most prominent value priorities gleaned from values histories and clinical experience, the brochure presents a spectrum of diverse but commonly heard health value statements within which patients can begin to locate their own values [28].

Akin to tiered consent for biobanking, Wish 2 (table 1) lists treatment choices relevant to many critical or endof-life cases. However, unlike tiered consent, this information is provided within the broader context of the patient's values and goals described in Wishes 1, 3, 4, and 5 (table 1). Thus, a patient is able to describe her wishes for care in a holistic manner despite the brevity, providing a surrogate more information than if a particular treatment choice were presented without context. This allows the surrogate to weigh the patient's treatment choice (which may be more or less directly relevant in a future scenario) along with his or her other values to determine the appropriate decision.

In addition to the expansion from specific choices to broader values, The Five Wishes also encapsulates the second shift: from a static document to a communicative process [21]. The Five Wishes urges conversation between patients, surrogates, wider families, and health professionals. It includes instructions for how to change or update an advance directive legally. Many other advance directive documents now follow The Five Wishes by casting themselves as decision aids designed to elicit major values and goals through ongoing multiparty communicative processes.

The advance directive solutions presented by values histories and The Five Wishes suggest that we look for analogous solutions to the epistemic and practical challenges facing biobanks. We propose a conceptual framework that uses (1) values histories as a model for biobank community engagement, (2) The Five Wishes as a model for biobanking individual decision aids, and (3) an itera- 
tive approach that encourages the information elicited from communities and individual donors to influence both each other and biobank governance as a whole.

\section{Implementation}

Values Histories for Biobank Community Engagement While it may be impracticable for biobanks to elicit extensive values histories from each individual donor, we suggest using the model of values histories to shape engagement with target communities. Community engagement modeled on values histories would create discursive space for eliciting broad values statements and narratives regarding goals of research, coupled with discussion reallife research examples, as central tasks.

Just as clinical patients are asked to place their preferences along a spectrum of treatment options and value priorities, communities can discuss their comfort with biobank activities on the spectrums of privacy, data sharing, and control options within the context of the values that the community holds. Just as the question about the level of trust that patients have in various decision-makers (like doctors, family and friends), communities can be prompted to discuss their levels of trust and distrust in various potential decision-makers that would influence biobank governance, such as healthcare institutions, governments, local service organizations, etc. Storytelling and the relationship between options and values could be explored.

Like clinical values histories, the shift would be from a primary focus on a discussion of discrete biobank options to a broader discussion of the outcomes and impacts that are most important to communities. This particular type of community conversation could reveal hopes and fears, shared consensuses, divisive issues, and a range of potential policy guidelines in ways that specifically asking for preferences among currently existing options could miss. Further, if different biobank policy opportunities and challenges emerge in the future, which is inevitable, the broader understanding of community priorities would help make informed decisions that would be in line with these preferences.

One specific goal of community engagement would be to inform the range of articulated values and research examples that will become the prompts in more streamlined decision aids for individual donors. Values that are most commonly stated, or most in tension with each other, as well as research examples that garner the most consensus or the most controversy in community values histories might be especially useful for framing decision aids. In this way, the time-consuming effort of the minority who participate in community engagement becomes a resource for the wider pool of individual recruits. One benefit of this suggestion is that it does not require a new allocation of resources or time, but rather refocuses types of community engagement that are already occurring in many biobanks.

In North America, one of the biggest efforts to engage communities around the issues of biobanks has been made by the Electronic Medical Records and Genomics Network (e-MERGE), an NIH-funded research consortium supporting genome-wide association studies (GWAS). Participating institutions in the e-MERGE network conducted a variety of public engagements probing attitudes toward wide-scale genomic research among target populations while introducing research aims of specific sites [29]. Member institutions include pioneers of community engagement on biobank policy such as the Mayo Clinic, Marshfield Clinic, Group Health Cooperative/University of Washington, and Vanderbilt University. They have used results of these community conversations to inform biobank policies on issues such as when reconsent is necessary, and to simplify educational materials and consent processes in ways that enable decisionmaking among individual recruits [29].

Another example of community engagement by biobanks is the Michigan BioTrust - a biorepository of de-identified neonatal bloodspots leftover from newborn screening. Through an NIH-funded partnership between the University of Michigan and Michigan State University, communities have been engaged through social media, town hall meetings, and a citizen's deliberative process $[30,31]$. These engagements also focused on specific biobank options and preferences, and resulted in modifications such as the publication of lay study descriptions and the addition of community members on the board.

The content of these engagement conversations tend to struggle with the same challenges that face individual research consent: namely, to the extent that they focus on disclosure of information about biobanks they are presupposing that they can provide adequate information about future unknown biobank decisions to the community, which they cannot. To the extent that they elicit input from communities, they tend to focus on either specific input on choices that the biobank currently faces (how to constitute a community advisory board, what sort of privacy safeguards should be put in place) or hypothetical future choices (commercialization decisions). In the former case, the community input may be useful 
Table 2. The Five Wishes adapted for biobanking

\begin{tabular}{|c|c|c|}
\hline \multicolumn{2}{|c|}{ Wish } & \multirow{2}{*}{$\begin{array}{l}\text { Biobank adaptation } \\
\text { 'Who should make decisions about my samples when I can't?' } \\
\text { - Ask donors whom they trust to represent them in the biobank and } \\
\text { the extent of their powers. } \\
\text { - Desirable and undesirable representatives, extent of representation; } \\
\text { characteristics of governance that they would trust to make decisions }\end{array}$} \\
\hline 1 & $\begin{array}{l}\text { 'Who should make decisions when I can't?' } \\
\text { - Prompts patients to choose a surrogate and define the extent } \\
\text { of their powers. } \\
\text { - Advises patients about desirable or undesirable characteristics in } \\
\text { surrogates. }\end{array}$ & \\
\hline 2 & $\begin{array}{l}\text { 'What treatments do I want?' } \\
\text { - Provides checklists of frequently heard general values statements } \\
\text { with items that can be checked, crossed-out, or supplemented. }\end{array}$ & $\begin{array}{l}\text { 'What uses do I want my samples used for?' } \\
\text { - Checklist of prevalent research goals and limitations from values } \\
\text { history in initial or ongoing community engagements. } \\
\text { - Iconic research scenarios identified through community engagement. }\end{array}$ \\
\hline $3-5$ & $\begin{array}{l}\text { 'How comfortable I want to be.' } \\
\text { 'How I want people to treat me.' } \\
\text { 'What I want my loved ones to know.' } \\
\text { - Elicits statements of relevant nonmedical values. } \\
\text { - Examples: quality of life, respect, communication. }\end{array}$ & $\begin{array}{l}\text { 'What do I want to protect about myself or my community?' } \\
\text { 'What do I want research to accomplish for me or my community?' } \\
\text { 'What do I want to ensure is a part of my relationship with the biobank?' } \\
\text { - Elicit statements of relevant nonresearch values. } \\
\text { - Examples: privacy, stigma, group harm, conflict with values, return } \\
\text { of results, economic/commercialization concerns. }\end{array}$ \\
\hline
\end{tabular}

now, but does not prepare the biobank to make future unforeseen decisions in light of this input. In the latter case, it is difficult if not impossible to predict what future scenarios may arise. A final question is how precisely to incorporate the values and views elicited from the community into individual consent documents.

While the intentions and the format of current community engagement is laudable, we believe that embracing the analogy with advance care planning and values histories in particular can provide helpful solutions to these challenges. The focus groups, town hall meetings, or public deliberative processes that are currently being implemented could be focused on locating community biobank preferences within the context of broader community values.

One potential example of this type of engagement occurred in the formation of a biobank in Western Australia. Deliberative public engagement was conducted there focused on specific preferences in the context of broader values [32]. The conversations with the public were split into 2 parts; in the first part of the engagement, participants discussed their broad hopes and concerns regarding the biobank, and then in the second part, they focused on specific recommendations for public policy. Like this, incorporating a values history framework into current and future community engagement approaches would not require a 'sea change' in engagement techniques, but rather a honing of its content and goals. Understood as a values history, community engagement could become a pivotal linchpin between the advance directives of indi- vidual donors and the surrogacy decisionmaking processes of biobank governance.

\section{The Five Wishes for Individual Permission}

The information gathered through a community values history could be used to inform biobank governance directly as well as to shape the content of decisionmaking aids that guide 'permission processes' for individual donors in particular communities ${ }^{2}$. Ideally such tools would be short, accessible and interactive - such as The Five Wishes in clinical contexts. The goal of each of the 5 wishes in the clinical context can be adapted to elicit a parallel value in the biobank context (table 2).

Wish 1: Who Should Make Research Decisions when I Can't?

Asking this question would serve as a clear disclosure that biobanks will be making decisions about their samples in the future that donors will not control. It presses biobanks to briefly articulate what governance structures they have for donor representation. In addition, this question provides guidance to those who are setting up biobank governance and must determine which community representatives to incorporate.

\footnotetext{
2 Even if a biobank decides that an opt-out permission process is acceptable, it is still not safe to assume that the policy decisions gleaned from community engagement are appropriate for individuals within that area. Biobanks can use information from community engagement for disclosure materials as well as to shape permission processes.
} 
Past biobanking public engagements have shown that potential donors want community representation in governance [33]. This advice has been taken to heart by several progressive biobanks. For example, the Michigan Biotrust for Health has a 'Community Values Advisory Board' and the Mayo Clinic Biobank has a 'Community Advisory Board'. By asking individuals who they trust to represent them, biobanks will be able to ensure that the members of such community advisory boards are truly representative and trusted by the donors to their biobank. As demographics and relationships shift, these members could shift as well.

Wish 2: How Do I Want My Samples Used?

As with the clinical Five Wishes, this section would revolve around articulating the donor's biggest goals for research and values related to research, rather than merely around highly specific research categories or methodologies. The ranges of values identified in the community values history, as well as the example research scenarios that came to organize discussion in that public engagement, would organize donor reflection and assessment of comfort levels and limits. While checklists from community engagement would allow quick circling or check-offs, write-in options would allow for expansion or idiosyncratic views.

\section{Wishes 3-5}

Wishes 3-5 in the clinical Five Wishes are aimed at eliciting relevant nonmedical values from patients. Similarly, biobanks should recognize that many individuals have values that may conflict with or override the values they hope to achieve from research. Past biobank community engagement has shown that issues of privacy, insurance or other discrimination, religious or cultural beliefs around the status of blood and the body, stigma, and commercialization are often value-laden in ways that vary greatly by community and individual. Likewise, different nations and states have different protections in place against some of these concerns, so these issues are likely to vary geographically. Specific questions to elicit nonresearch values would be informed by actual community values histories, but might include questions such as:

- What do I want to protect about myself and my community?

- What community goods are more important to me than progress in health research?

- What are the most important qualities in my relationship with my community?

\section{Closing the Loop: Interactions between Values Histories, Decision Aids and Governance}

A further benefit of this model is that it provides more clear guidance on how different levels of engagement can be used together. Decision aids shaped by community values histories could help individual recruits benefit from public engagement and make authentic decisions about whether to give permission. At the same time, when aggregated, decision aids of those who donate (and even of those who decline) could provide relevant data for biobank surrogate decisionmaking in governance decisions. Decision aids could thereby become a new form of community engagement. Aggregated information from decision aids could help the biobank to realize what studies would likely generate consensus or controversy among donors, when special protections may be needed for identifiable minorities, etc. and when new community engagement or even individual discussions with donors should occur. All this results from the recognition that neither engagement at the community nor the individual level constitute 'informed consent', since this is not possible for biobanks. Rather, they are ways of building trust and eliciting information in a way akin to the relationship and communication between a patient and her designated surrogate. The goal is not to fulfill a legal consent transaction, but rather to reach a level of familiarity such that the biobank can be entrusted to make research decisions in the future on the donors' behalf.

\section{Limitations}

While individual decision aids informed by community values histories have much to offer to both biobanking permission processes and biobank surrogate decisionmaking, their limits should be explicitly recognized. In the context of current practice, if biobanks are already committed to using blanket permission, they should make clear that individual values expressed in the aid will not be authoritative in decisions about particular uses of samples. This could be acknowledged while utilizing decision aids and community engagement in determining donor comfort level and gaining a sense of the overall ethos of the donor pool.

Even biobanks that intend to give decision aids (either individually or in aggregate) authority should still admit the surrogate challenge involved in trying to make decisions authentic to the donor by what is revealed through the process. The improvements gained from shifting
Solomon/Mongoven 
from individual decisions to broader values do not solve the underlying problem that no decision aid can anticipate the future decisions faced by a biobank, nor the future discrete choices preferred by a donor. Limits aside, to say the biobank will do its best to make decisions in line with the values expressed by the donor is more honest than pretending to give the donor straightforward agency through a tiered consent process that denies epistemic limits.

\section{Conclusion}

Our conceptual model proposes creative adaptation from the history of advance directives in clinical medicine to transform biobank practice. Biobank community engagements can be envisioned as community values histories. Community values histories can then shape the development of decision aids for individual donors that incorporate parameters from community engagement. Such decision aids should be streamlined and user friendly such as The Five Wishes in clinical contexts. Data from individual decision aids can then be aggregated to inform the governance processes through which biobanks execute their surrogate decisionmaking function. Aggregated data from individual decision aids then becomes another form of public engagement, bringing the communicative process full circle.

While many biobanks are developing community engagement practices that implicitly embody insights of our model, the particular aims of these engagements often remain varied and ambiguous. There is often not a clear theoretical or practical connection between the information obtained from community engagement, the development of options or forms for individual donors, and biobank decisionmaking about what research to support with whose samples. Even with increasing community engagement, many biobanks remain 'stuck' offering either blanket or tiered consent options for donors despite the problems with both. Unless eliciting values becomes the focus of conversation, engagement is unlikely to resolve the fundamental challenge that biobanks face: applying current views, from the community or the individual, to unknown future scenarios.

No permission process can remove the epistemic limits that make 'informed consent' a misguided ideal for biobanking. Even in cases of unusual donor attentiveness, such as among disease advocacy groups, actual future research remains unknown. Progressive systems themselves create epistemic limits, such as participant over- load or fatigue in the face of a barrage of information or loss of initial novelty.

Approaches from advance directives should be adopted by biobanks because their starting point is the recognition of epistemic limits. Biobank engagement with donors should refocus on values, elicited in communicative processes that connect community engagement to development of individual decision aids. Then both community and individual-level engagements can inform biobank governance and surrogate decisionmaking. If this conceptual extrapolation could be accomplished flexibly enough to be adaptable to a variety of biobanking contexts and simply enough to become widely used, biobanking permission would become more robust and biobanks would have more concrete bases for their surrogate role.

A conceptual shift to the analogy of clinical advance directives sheds new light on well-known challenges of communication and consent in biobanking. It suggests new ways of evaluating emerging innovative solutions. A strength of our approach is that practices akin to it are already being used by progressive biobanks, albeit without the support of a conceptual rationale or a consistent model.

\section{Acknowledgements}

The authors gratefully acknowledge the National Institutes of Health for 2 federal grants that supported this work: S.S. is supported by the CTSA grant UL1 RR024992 from the NIH National Center for Research Resources, and A.M. is supported by 1R01HD067264-01 from the NIH Eunice Kennedy Shriver National Institute of Child Health and Human Development.

The authors gratefully acknowledge the American Society of Bioethics and Humanities and relevant members who attended an oral presentation, piloting ideas elaborated in this paper at the Society's annual meeting, Washington D.C., October 2012. The paper is indebted to several questions and comments offered there.

\footnotetext{
References $\nabla_{1}$ Mongoven AM, Solomon S: Biobanking: shifting the analogy from consent to surrogacy. Genet Med 2012;14:183-188.

2 Sheehan M: Can broad consent be informed consent? Public Health Ethics 2011;4:226-235.

3 Allen J, McNamara B: Reconsidering the value of consent in biobank research. Bioethics 2011;25:155-166.

4 Genetic Alliance: Reg4ALL ${ }^{\circledR}$. https://www. reg4all.org/ (accessed April 24, 2014).

5 Rhodes R, Holzman I: Surrogate decision-making: a case for boundaries; in Thomasma DC, Weisstub DN (eds): The Variables of Moral Capacity. Netherlands, Springer, 2004, pp 173174.
} 
6 Brudney D: Beyond autonomy and best interests. Hastings Cent Rep 2009;39:31-37.

7 Welie JV: Living wills and substituted judgments: a critical analysis. Med Health Care Philos 2001;4:169-183.

8 Gready RM, Ditto PH, Danks JH, Coppola KM, Lockhart LK, Smucker WD: Actual and perceived stability of preferences for life-sustaining treatment. J Clin Ethics 2000;11:334346.

-9 Danis M, Garrett J, Harris R, Patrick DL: Stability of choices about life-sustaining treatments. Ann Intern Med 1994;120:567-573.

10 Carmel S, Mutran EJ: Stability of elderly persons' expressed preferences regarding the use of life-sustaining treatments. Soc Sci Med 1999;49:303-311.

-11 Tilden VP, Tolle SW, Nelson CA, Fields J: Family decision-making to withdraw life-sustaining treatments from hospitalized patients. Nurs Res 2001;50:105-115.

-12 Abbott KH, Sago JG, Breen CM, Abernethy AP, Tulsky JA: Families looking back: one year after discussion of withdrawal or withholding of life-sustaining support. Crit Care Med 2001;29:197-201.

13 Wright AA, Zhang B, Ray A, Mack JW, Trice E, Balboni T, Mitchell SL, Jackson VA, Block SD, Maciejewski PK, Prigerson HG: Associations between end-of-life discussions, patient mental health, medical care near death, and caregiver bereavement adjustment. JAMA 2008;300:1665-1673.

14 Brown BA: The history of advance directives. A literature review. J Gerontol Nurs 2003;29: 4-14.
15 Drabiak-Syed K: Lessons from Havasupai Tribe v. Arizona State University Board of Regents: recognizing group, cultural, and dignity harms as legitimate risks warranting integration into research practice. J Health Biomed L 2010;6:175.

16 Munsterhjelm M: Living Dead in the Pacific: Contested Sovereignty and Racism in Genetic Research on Taiwan Aborigines. Vancouver, UBC Press, 2014.

17 Kozlakidis Z, Cason RJ, Mant C, Cason J: Human tissue biobanks: the balance between consent and the common good. Res Ethics 2012;8:113-123.

18 Emanuel LL, Emanuel EJ: The medical directive: a new comprehensive advance care document. JAMA 1989;261:3288-3293.

19 Lemke AA, Wolf WA, Hebert-Beirne J, Smith ME: Public and biobank participant attitudes toward genetic research participation and data sharing. Public Health Genomics 2010; 13:368-377.

20 Simon CM, L'Heureux J, Murray JC, Winokur P, Weiner G, Newbury E, Shinkunas L, Zimmerman B: Active choice but not too active: public perspectives on biobank consent models. Genet Med 2011;13:821-831.

21 Sabatino CP: The evolution of health care advance planning law and policy. Milbank Q 2010;88:211-239.

22 Lambert P, Gibson JM, Nathanson P: The values history: an innovation in surrogate medical decision-making. J Law Med Ethics 1990; 18:202-212.

23 Doukas DJ, McCullough LB: The values history: the evaluation of the patient's values and advance directives. J Fam Pract 1991;32:145153.

-24 Grundstein-Amado R: Narrative inquiry: a method for eliciting advance health care directives. Humane Med 1992;8:31-39.
25 Prendergast TJ: Advance care planning: pitfalls, progress, promise. Crit Care Med 2001; 29(suppl 2):N34-N39.

26 Hawkins NA, Ditto PH, Danks JH, Smucker WD: Micromanaging death: process preferences, values, and goals in end-of-life medical decision making. Gerontologist 2005;45:107117.

27 Five Wishes. http://www.agingwithdignity. org/forms/5wishes.pdf (accessed August 13, 2013).

28 Aging With Dignity Five Wishes. http://www. agingwithdignity.org/five-wishes-resources. php (accessed April 25, 2014)

29 Lemke AA, Wu JT, Waudby C, Pulley J, Somkin CP, Trinidad SB: Community engagement in biobanking: experiences from the eMERGE Network. Genomics Soc Policy 2010;6:35-52.

30 Langbo C, Bach J, Kleyn M, Downes FP: From newborn screening to population health research: implementation of the Michigan BioTrust for health. Public Health Rep 2013; 128:377-384.

31 Mongoven A, McGee H: IRB review and public health biobanking: a case study of the Michigan BioTrust for Health. 2011. http:// philpapers.org/rec/MONIRA-3 (accessed April 24, 2014).

32 Molster C, Maxwell S, Youngs L, Potts A, Kyne G, Hope F, Dawkins H, O'Leary P: An Australian approach to the policy translation of deliberated citizen perspectives on biobanking. Public Health Genomics 2011;15: 82-91.

33 Trinidad SB, Fullerton SM, Bares JM, Jarvik GP, Larson EB, Burke W: Genomic research and wide data sharing: views of prospective participants. Genet Med 2010;12:486-495. 